\title{
Structural study of the inhibitory mechanism of tau recognition antibody to tau aggregation
}

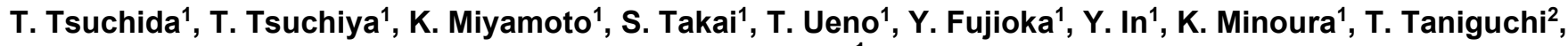 \\ K. Tomoo'
}

\author{
${ }^{I}$ Osaka Medical and Pharmaceutical University, Takatsuki, Osaka, Japan, \\ ${ }^{2}$ Pharmacrea Kobe Co. Ltd., Kobe, Hyogo, Japan
}

ompu72118051@s.ompu.ac.jp

\begin{abstract}
Alzheimer's disease (AD) is a neurodegenerative disease accompanied by the accumulation of misfolded proteins. AD pathology is characterized by the extracellular amyloid plaques and the neurofibrillary tangles (NFTs). NFTs consist of paired helical filament (PHF) and abnormally phosphorylated tau is known to form the PHF.

Tau protein that exists in the brain neuron cells is important for the stabilization and elongation of the microtubules. Tau contains a microtubule-binding domain (MBD) consisting of three or four repeats of about 30 similar amino acids (R1-R4). The MBD is not only important for the binding of tau to microtubule but also plays a key role for the abnormal self-aggregation of tau. Even though the sequences of repeat peptides of MBD are similar to each other, the ability of self-aggregation of these peptides is quite different. Especially, the short regions of both ${ }^{275} \mathrm{VQIINK}{ }^{280}$ and ${ }^{306} \mathrm{VQIVYK}^{311}$ that are the start sequence of R2 and R3 respectively have an important role for the tau self-aggregation.
\end{abstract}

Several therapeutic approaches targeting tau aggregation have been proposed, such as a tau aggregation inhibitor. Among tau aggregation inhibitors, a monoclonal antibody may be particularly effective because of its specificity to the target molecule. Thus, in searching for a tau aggregation inhibitor, we made a monoclonal antibody to tau (Tau2r3) using the ${ }^{272}$ GGKVQIINKKLD ${ }^{283}$ epitope peptide from the MBD in tau and prepared the Fab domain (Fab2r3) from Tau2r3. We analyzed the inhibitory function of Fab2r3 for tau aggregation and determined the tertiary structure of the Fab2r3 complex with VQIINK peptide.

The results of thioflavin S (ThS) fluorescence and TEM (negative-staining electron microscopy) measurement clearly showed that Fab2r3 inhibited the tau aggregation, and the inhibitory function of Fab2r3 seems to occur through specific binding to the VQIINK sequence by the isothermal titration calorimetry (ITC) analysis.

To elucidate this inhibition mechanism, we analyzed the tertiary structure of Fab2r3 and VQIINK peptide complex by X-ray crystallography. The VQIINK peptide makes many hydrogen bonds and two hydrophobic interactions with Fab2r3. Among these interactions, we supposed that the hydrophobic interaction has a key role for the antigen recognition of the Fab2r3.

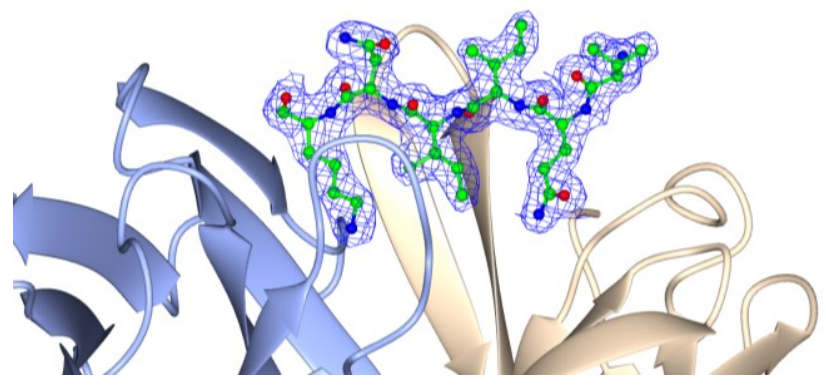

Fig.1 The Fab2r3-VQIINK peptide complex structure

[1] Tsuchida, T., Susa, K., Kibiki, T., Tsuchiya, T., Miyamoto, K., In, Y., Minoura, K., Taniguchi, T., Ishida, T. \& Tomoo, K. (2020). FEBS Letters, 594, 2140-2149

Keywords: tau, Alzheimer's disease, antibody, Fab domain 